\title{
Prostate Cancer Ultrasound: Is Still a Valid Tool?
}

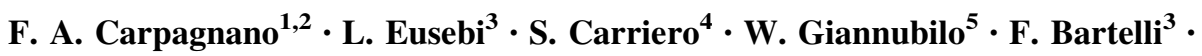 \\ G. Guglielmi ${ }^{1,2,6}{ }_{(\mathbb{D})}$
}

Accepted: 15 June 2021 / Published online: 9 July 2021

(C) The Author(s) 2021

\begin{abstract}
Purpose of Review The main purpose of this paper review is to highlight the latest ultrasound (US) imaging technologies of the prostate gland, an organ increasingly at the center of attention in the field of oncological diseases of the male sex, which needs a $360^{\circ}$ evaluation in order to obtain tailored therapeutic planning. Specialist urological evaluation is designated for this purpose, together with integrated prostate imaging which currently tends to focus more and more on the use of US imaging and its state-ofthe-art technologies in iconographic diagnosis, biopsy and, sometimes, treatment of prostatic cancer.

Recent Findings In particular, the main tools to which reference is made, represent a valid aid to basic US technologies already widely known and diffused, like the grayscale US or the Doppler US, for a "multiparametric" evaluation of the prostate cancer. The concept of multiparametricity is explained by the integration of prostate
\end{abstract}

This article is part of the Topical Collection on Geriatrics.

G. Guglielmi

giuseppe.guglielmi@unifg.it;

http://www.unifg.it

1 Department of Clinical and Experimental Medicine, Foggia University School of Medicine, Viale L. Pinto 1, 71121 Foggia, Italy

2 Radiology Unit, “Dimiccoli” Hospital, Barletta, Italy

3 Radiology Unit, “Carlo Urbani” Hospital, Jesi, Italy

4 Department of Radiology, Milano University School of Medicine, Milano, Italy

5 Urology Unit, "Carlo Urbani”" Hospital, Jesi, Italy

6 Radiology Unit, Hospital "Casa Sollievo Della Sofferenza", San Giovanni Rotondo, Foggia, Italy imaging obtained both with the US evaluation of the gland before and after administration of contrast medium, with the elaboration of parametric maps of quantitative measurement of the enhancement, and with elastography that provides information about the tissue consistency, a finding that strongly relates with the degree of cellularity and with the tumor grading.

Summary Prostate cancer screening consists of dosing serum levels of prostate-specific antigen (PSA) and performing digit-rectal examination (DRE), more or less associated with transrectal prostate ultrasound (TRUS). However, although these are the most common techniques in clinical practice, they have numerous limitations and make the diagnosis of prostate cancer often challenging. The purpose of mp-US is to enrich the clinical-laboratory data and, above all, the standard US imaging with further details to strengthen the suspicion of malignancy of a prostate tumor, which needs to be addressed to diagnostic deepening with biopsy. This review article provides a summary of the current evidence on mp-US imaging in the evaluation of a clinically significant prostate cancer, comparing the data obtained to the imaging of mp-MRI, the reference tool both in diagnosis and staging.

Keywords Mp-US · Prostate cancer - CEUS - SE - SWE

\section{Introduction}

It is now widely known that prostate cancer $(\mathrm{PCa})$ is the most common type of malignant cancer in the male population of Northern and Western Europe (>200 per $100,000 \mathrm{men} / \mathrm{year}$ ), geographical prevalence dictated by the numerous exogenous factors (e.g., nutrition or UV rays exposure) that contribute to cause prostate cancer together 
with individual predisposition, age, and familiarity [1, 2]. There are many factors influencing the survival of prostate cancer patients, in particular the extent of the tumor and/or the presence of distant metastases, which is why early detection is indispensable. Routine screening for patients at risk, by age or familiarity, currently involves the dosing of serum-specific prostatic antigen (PSA) and digit-rectal examination (DRE), in addition to any subsequent prostate imaging investigations [3]. In this regard, although screening is an indispensable tool for early diagnosis and treatment, these investigation techniques are not very specific and not very sensitive, and therefore, there is a risk of incurring clinically significant undiagnosed cancers or clinically insignificant but overestimated cancer [4, 5]. Therefore, it is important to identify the most appropriate imaging method in order to improve the detection of prostate cancer and, in recent years, multiparametric Magnetic Resonance Imaging (mp-MRI) seems to have a central role for this purpose [6]. However, mp-MRI also has many limitations, starting from the most common contraindications in the execution of MRI, up to those more specifically related to prostatic mp-MRI, such as endorectal coil discomfort, long duration of the examination, or difficulty in imaging interpretation [7••]. An easier imaging technique is represented by transrectal ultrasound (TRUS), particularly in conventional brightness-mode (Bmode) and Doppler mode, although grayscale ultrasound imaging does not show satisfactory results in detection of prostate cancer, in terms of sensitivity and specificity. In fact, the new frontiers of imaging are increasingly oriented towards more specific and cutting-edge ultrasound techniques such as contrast-enhanced ultrasound (CEUS) and elastography, currently known as multiparametric ultrasound techniques (mp-US) [8•], and potentially comparable to the dynamic contrast-enhanced (DCE) and diffusionweighted (DW) mp-MRI sequences, respectively [7••]. The purpose of this review article is to describe these new ultrasound techniques and to evaluate their practical usefulness as imaging tools in the early detection of prostate cancer, as well as performing a targeted prostate biopsy and treatment.

\section{Conventional TRUS: B-Mode and Doppler Ultrasound}

The ultrasound technique currently most common and familiar to many categories of medical specialists, mainly radiologists and urologists in our case, is represented by the classic B-mode grayscale TRUS, as a low cost and readily available method. However, it should be borne in mind that in many fields of application, ultrasonography has limited diagnostic accuracy and the challenge becomes even more difficult when it comes to distinguish between the multiple pathogenic noxae affecting the prostate gland. Prostatic lesions, in fact, either benign or malignant, can appear with different sonographic patterns to the TRUS imaging and the prostatic carcinoma, in particular, occurs in most cases as a hypoechoic nodule, compared to the surrounding glandular parenchyma (60-80\%) (Figs. 1A, 2, 3A), but there are as many cases in which it may appear as isoechoic (30-40\%) or hyperechoic (about 1.5\%) (Fig. 2A) [3]. It is, therefore, clear to understand how this imaging technique has a low specificity and sensitivity, especially in the distinction between a prostatic lesion and the normal parenchyma and even more if it is a lesion that affects the transitional zone (TZ), considering the heterogeneity of sonographic patterns of the latter [9].

There are, however, some collateral findings that, although not pathognomonic, in addition to direct visualization of a suspicious prostatic ultrasound image, can increase the suspicion of neoplastic pathology with malignant characteristics, such as asymmetry and irregularity of the gland in terms of morphology and echogenicity, capsular bulging, or the extension of the tumor's contact surface to the prostate capsule above $23 \mathrm{~mm}$ suggesting a likely extracapsular extension (ECE) [10•]. It should be noted that TRUS is by no means a reliable method for the locoregional staging of prostatic cancer, for which the use of mp-MRI is now widely used (Figs. 1, 2, 3, 4) [11].

In the field of ultrasound prostate imaging, the frequency and shape of the transrectal probe play a very important role. It has been noticed, in fact, that the high frequencies, typically used in linear probes, are able to provide a better spatial and temporal resolution but, at the same time, are associated with a greater attenuation of the ultrasound beam.

The use of micro-convex arrays, although they require greater interpolation between lines forming the ultrasound image and have a lower spatial resolution than linear probes if positioned at the tip of the probe (end-fire probe) or proximally (biplane transducer) and if used at mediumhigh frequencies (7-9 MHz), seems to be of particular diagnostic aid in ultrasound imaging of the prostate gland $[8 \cdot]$.

Added value to grayscale ultrasound imaging is provided by the techniques of Color Doppler US (CDUS) and Power Doppler US (PDUS) that allow to detect more prostate cancers than grayscale TRUS alone (Figs. 1B, 2B) [12, 13]. A Cheng and Rifkin retrospective analysis of 619 patients showed an increase of about $12 \%$ in prostate cancer cases diagnosed with CDUS not visible with the classic grayscale B-mode [14].

Three different flow patterns with CDUS can be distinguished from the suspected prostate lesion, represented 

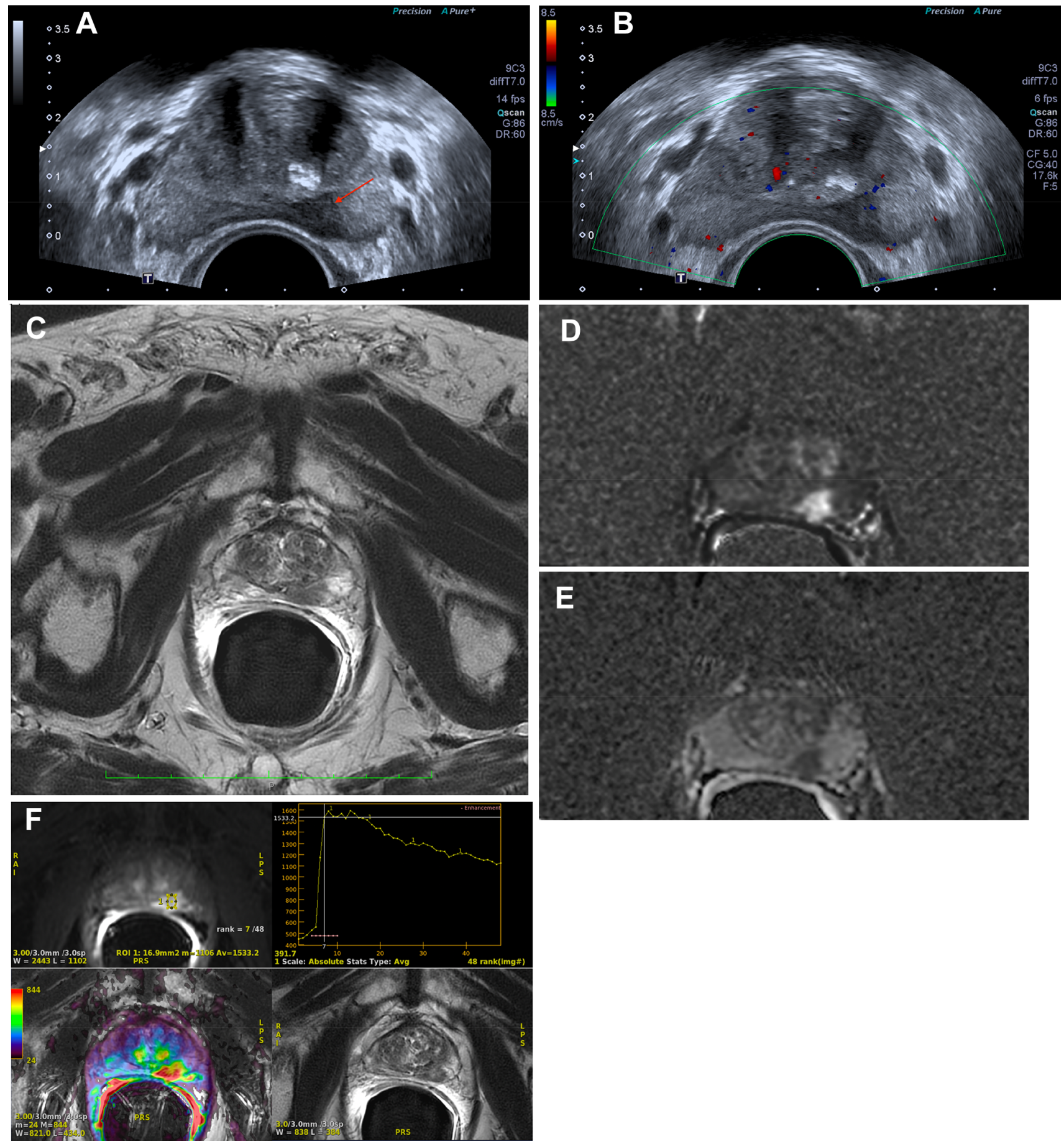

E

Fig. 1 Red arrow (A) shows an irregular round-shape nodule affecting the peripheral zone (PZ), with bulging of prostate capsule on left side, in a slightly symptomatic 78 years-old patient. It has a typical hypoechoic appearance on grayscale US and a not significantly increased blood flow at Doppler US (B). The lesion is more clearly seen on mp-MRI, first on the axial plane in the morphological T2-weighted sequence $(\mathbf{C})$, then with a restricted diffusion on DWI

(D) and ADC map (E) represented, respectively, by high-intense and low-intense signals. Parametric map (F) generated from dynamic contrast enhancement (DCE) evaluation of the PCa highlights the suspected area with red code, standing out from the normal prostatic parenchyma, with an early intense hyperenhancement, related to increased tumor vascularity, followed by a rapid wash-out (type 3 curve) (Color figure online) 

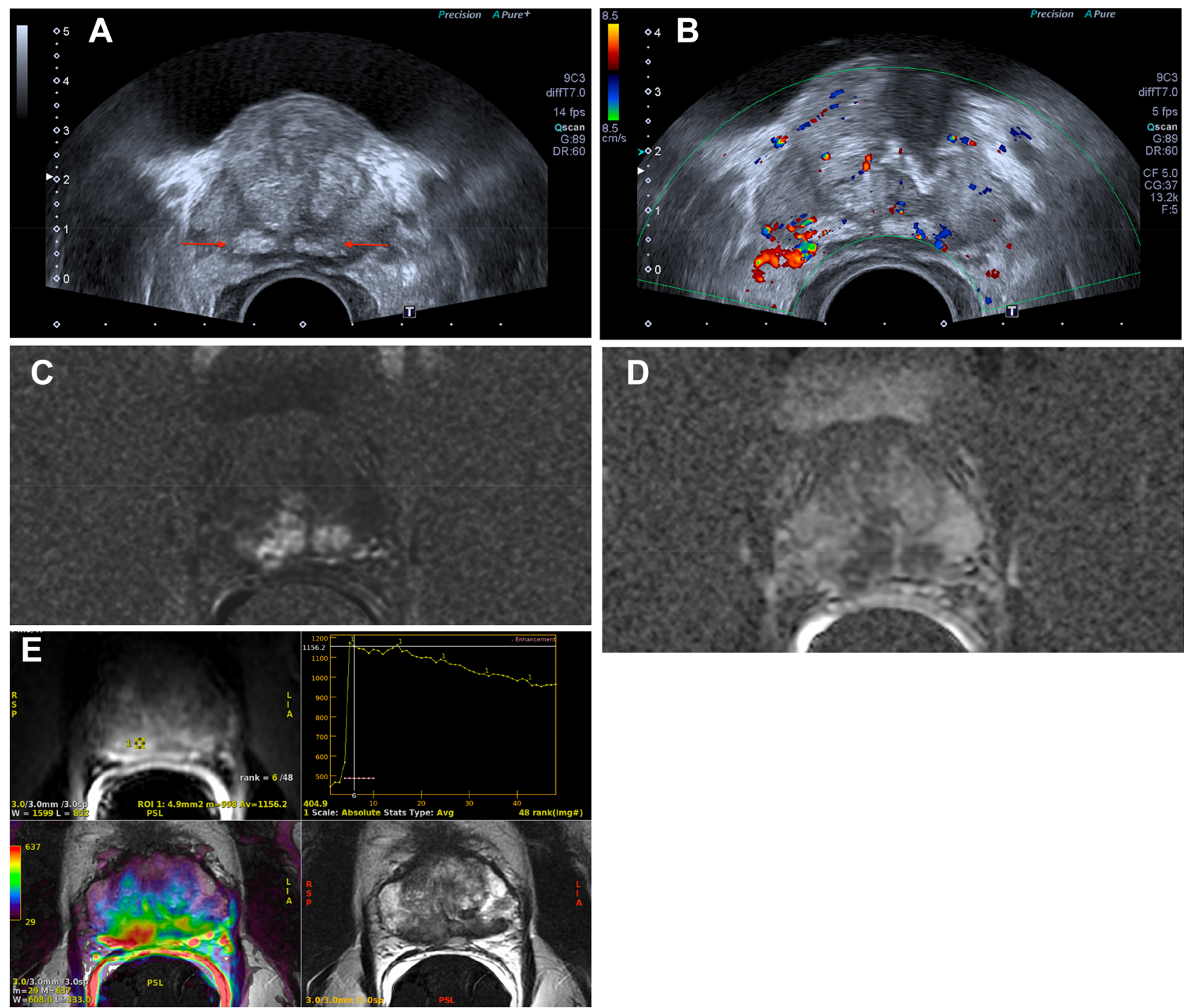

Fig. 2 68-year-old man affected by mid-gland bilateral peripheral zone (PZ) prostate cancer (red arrows), appearing as two oval-shape hyperechoic nodules at grayscale US imaging (A), with blood flow disturbance seen at conventional Doppler imaging (B). The patient underwent mp-MRI which confirmed the presence of two prostatic nodules of PZ with slight extracapsular extension (ECE), showing restricted diffusion on diffusion-weighted imaging (DWI) and apparent diffusion coefficient (ADC) map (high-signal intensity in $\mathbf{C}$ and relevant low-signal intensity in $\mathbf{D})$. The analysis of

by surrounding, focal or diffuse flux, the latter representing the most frequent category.

Although not all tumors are hyper-vascular (Fig. 3B) and not all hyper-vascular lesions are malignant, several recent studies have focused on neo-angiogenesis and increased blood flow of malignant lesions as key elements in the usefulness of Doppler imaging [15]. Thanks to the possibility of obtaining a corresponding histological examination, after transrectal biopsy, for a certain diagnosis, it was possible to obtain values of sensitivity and

enhancement's kinetics (E) within the suspected prostate regions showed an early intense enhancement of both nodules, visible either on the colorimetric map generated from dynamic contrast enhancement (DCE) evaluation (note the suspected areas are coded in red), either on a "type 3" signal-time intensity curve. Final histological evaluation confirmed a high-grade prostate cancer. The iconography shows a semi-quantitative evaluation of only one of the two suspected prostatic nodules (the largest), marked with a ROI (region of interest) (Color figure online)

specificity of the Doppler US technique, respectively, of $81 \%$ and $68 \%$, with slightly higher sensitivity values for PDUS [16]. In particular, some studies suggest that there is a directly proportional correlation between the degree of vascularization of prostatic cancer and the Gleason score [12].

According to a study by Halpern et al., an adverse potential aspect of CDUS and PDUS techniques could be related to a physiological asymmetry of prostatic blood flow, which would appear to be greater in the left lobe due 
to the homolateral decubitus position most frequently used during ultrasound examination. These assumptions have been confirmed by an increased number of prostate biopsies performed in the left portion of the gland, due to increased blood flow seen at Doppler US, and not associated with a corresponding and agreed histological examination [17].

\section{Contrast-Enhanced Ultrasound (CEUS)}

Contrast-enhanced ultrasound (CEUS) is a recent onset technique that is nowadays being used in numerous specialist fields and in diagnostic imaging. It is based on the intravenous administration of 1-10-mm-diameter gas-filled microbubbles (less than a red blood cell), encapsulated in a lipid or surfactant envelope, used as a contrast medium thanks to their ability to reach the capillary bed, in view of its small size, and thus provide information on tissue micro-perfusion [18]. While the effectiveness of the ultrasound contrast medium may appear to be comparable to, or at times less than, the contrast media used in other imaging techniques such as computed tomography (CT) and magnetic resonance imaging (MRI), on the other hand, it should be noted that the tolerance and handling are higher than those [19]. By contrast, in fact, there are far fewer adverse reactions than gadolinium and/or iodine contrast media. Moreover, if we consider that the gas-filled
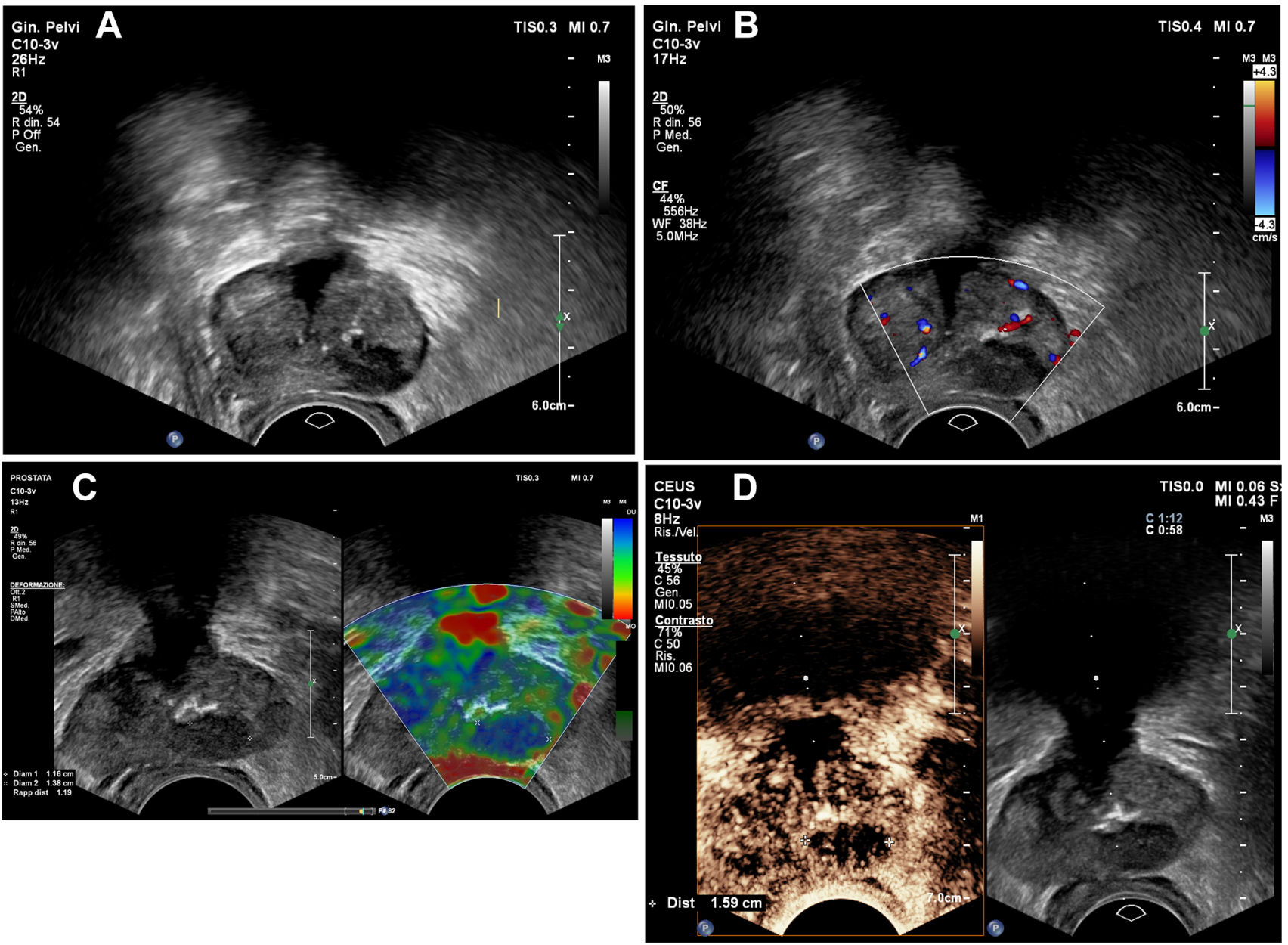

Fig. 3 A 75-year-old man affected by prostatic adenocarcinoma affecting the left peripheral zone (PZ), appearing as an irregular hypoechoic round-shape nodule on grayscale TRUS (red arrow in $\mathbf{A}$ ) and not showing an increased blood flow at Doppler imaging (B). The entire gland was then evaluated by performing a Strain Elastography (SE) and contrast-enhanced US (CEUS) acquisition: the colorimetric map $(\mathbf{C})$ obtained from SE examination shows the suspected nodule encoded in blue, thus, explaining the more rigid and less deformable

tissue's nature, confirmed also by the CEUS acquisition performed on the arterial phase (D) and the contrast acquisition curves $(\mathbf{E})$ of the neoplastic lesion (blue) compared with those of the apparently benign adjacent gland (orange). A T2-weighted mp-MRI axial acquisition (F) together with DWI $b=1600(\mathbf{G})$ and correspondent ADC reconstruction $(\mathbf{H})$ has been used as a comparison tools to confirm what resulted from mp-US (Color figure online) 

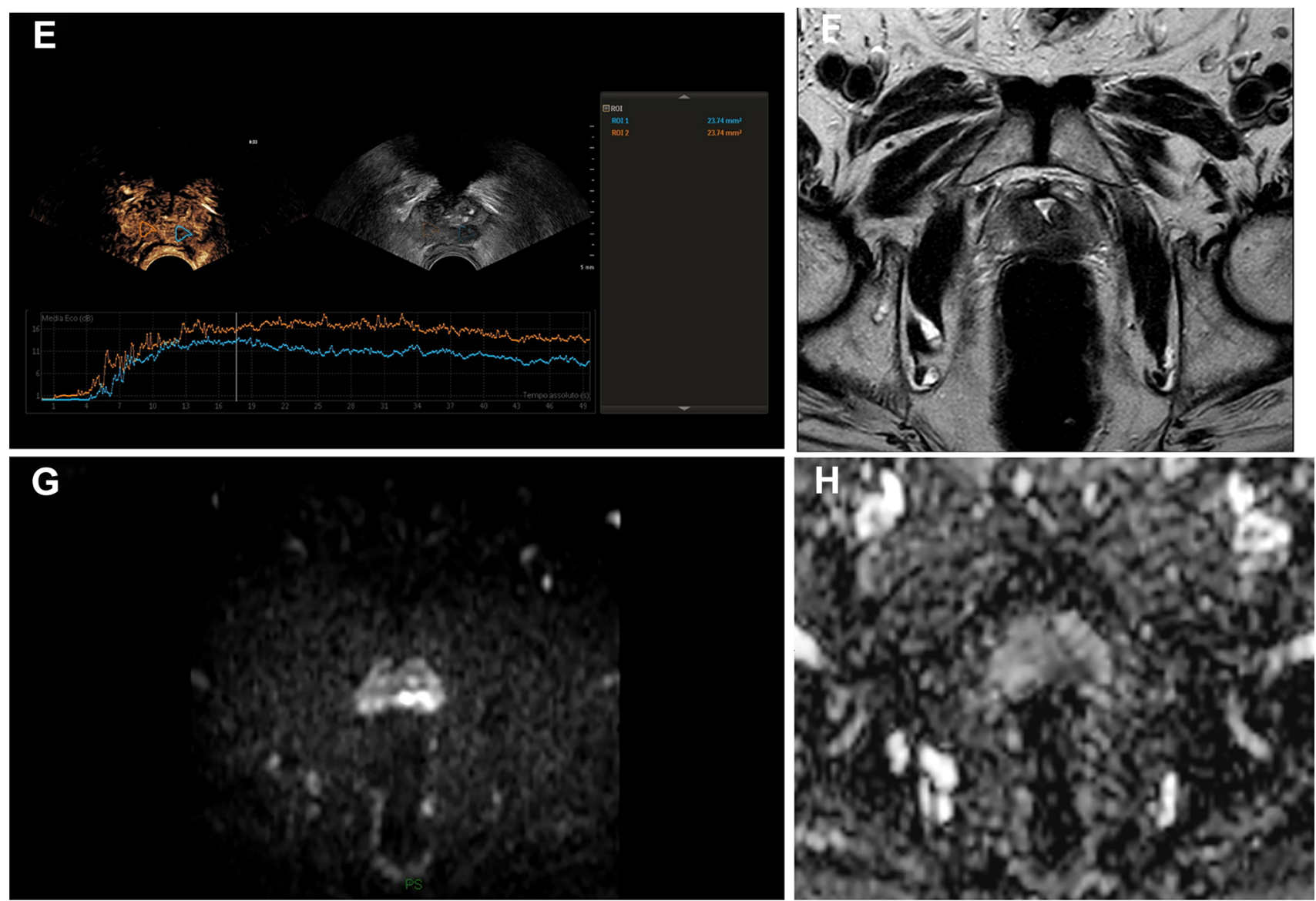

Fig. 3 continued

microbubbles do not reach the renal district and the urinary tract, it is clear how the problem of the potential nephrotoxicity of contrast media passes in the background: they can be used regardless of the kidney function of patients and/or the presence of urinary tract obstruction [20]. This ultrasound contrast medium property finds an additional point in its favor since, being a pure blood pool agent, it directly reaches the vascular district without spreading into the interstice of the surrounding tissues, element that could obscure the micro-vascularization, thus, providing a better evaluation of blood microflows than CDUS [21].

If, therefore, the principle on which CEUS is based is precisely that of highlighting micro-vascularization, thus, providing essential additional information to simple US grayscale images, it is easy to understand how a neoplastic lesion with a thriving neovascularization process is a preferred target of this imaging technique. A growing prostate cancer, in fact, compared to the portion of healthy contralateral gland, is commonly characterized by a higher density of micro-vessels of irregular appearance and distribution when compared with the linear orientation of the vessels, from the periprostatic neuro-vascular bundle, which supply the glandular parenchyma [22]. A metaanalysis by $\mathrm{Li}$ et al. conducted on 16 recent studies and a total of 2.624 patients, shows the added value of CEUS compared to normal grayscale ultrasound, reporting sensitivity and specificity values of $70 \%$ and $74 \%$, respectively, in identifying prostate cancer [23].

Prostatic CEUS is performed by using a transrectal probe with dedicated post-contrast sequences with low acoustic power, as well as low mechanical index values, allowing detection of microbubbles by the ultrasonic beam thanks to their curvilinear interface [8•]. The procedure starts with the identification of the suspected glandular area by any ultrasound technique (B-mode or CDUS, for example) (Fig. 3A, B) and then completed by the administration of ultrasound contrast medium keeping the transducer focused on the area of interest and activating the ultrasound mode with low acoustic power (Fig. 3D). So, what is the advantage? One might think that a simple grayscale ultrasound with the aid of Doppler mode is sufficient to identify the prostate lesion candidate for biopsy, but with how much accuracy? Several studies have shown that the use of the ultrasound contrast medium has greatly 
improved the identification of hyper-vascularized $\mathrm{PCa}$ nodules compared to Doppler US, increasing specificity from 79 to $87 \%$ and sensitivity from 54 to $93 \%$, respectively [24]. It seems clear, therefore that in this way, it is possible to carry out a more refined selection of the nodules candidated for biopsy, without, however, being able to say that the CEUS is able to avoid the biopsy examination, which remains, however, the key passage for the diagnosis of certainty.

This theory becomes even more consistent if we consider the high number of false positives related to inflammatory prostatic conditions or benign prostatic hyperplasia $(\mathrm{BPH})$, which cause an increase in vascularity as well as the size of the gland, especially the transition zone (TZ)
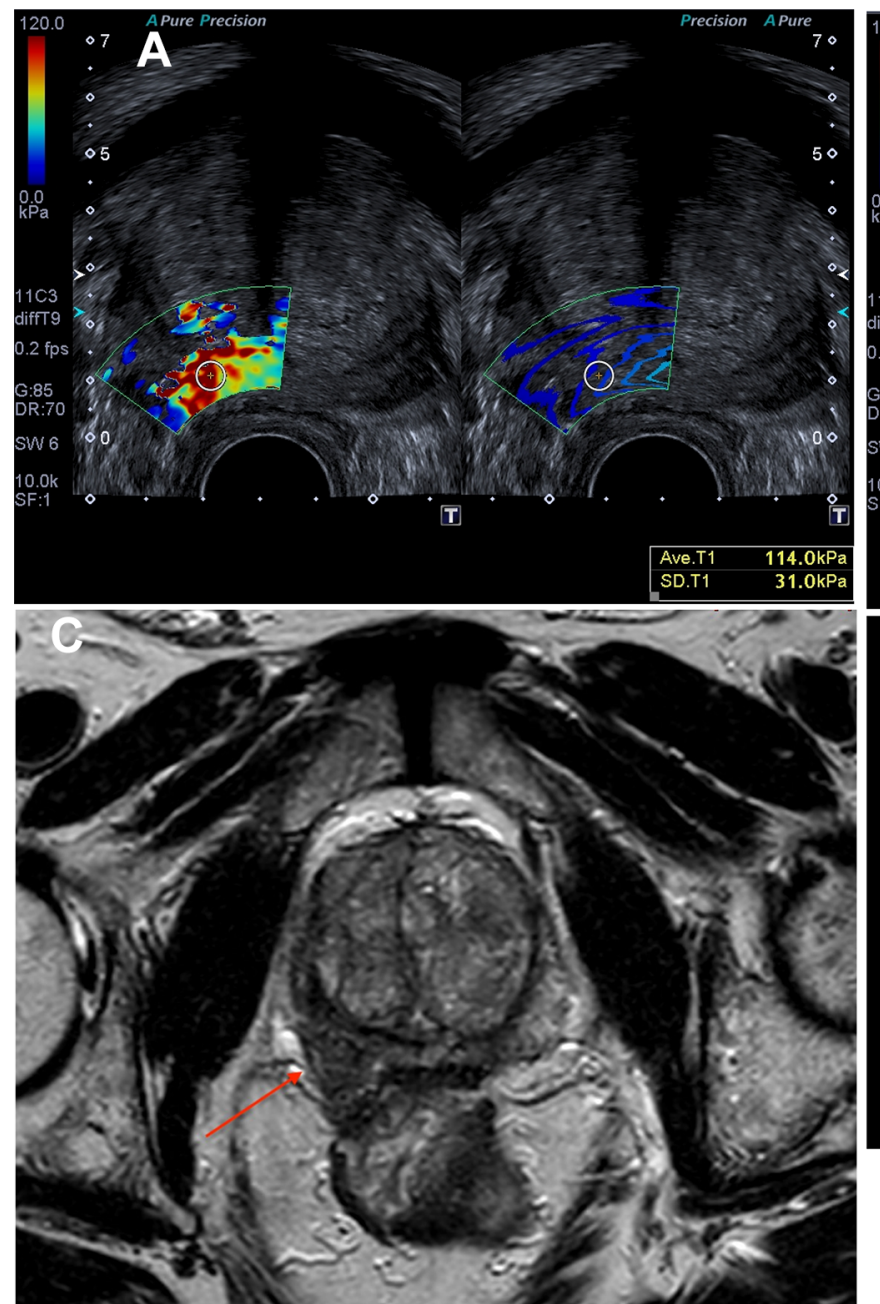

Fig. 4 Incidental discovery of a prostatic nodule affecting the right posterior peripheral zone (PZ), corresponding to a Gleason $4+4 \mathrm{PCa}$ in a 73-year-old man. A shows an increased stiffness (ROI: Average $114.0 \mathrm{kPa}$ ) of the prostatic adenocarcinoma measured by SWE, which allowed a direct measurement of tissue rigidity visually represented by a red area on the colorimetric map (the elastogram), superimposed on the B-mode ultrasound images, in contrast to the more elastic boarding tissue encoded in blue (B). The lesion, before going on and may obscure the flow associated with the presence of a malignant lesion.

However, in addition to vascular asymmetry, another aspect evaluable with CEUS is the quantitative measurement of blood flow of the suspected prostatic nodule due to the possibility of measuring contrast uptake over time, by means of dedicated software similar to those used in MR imaging, and to derive intensity-time curves (TIC) (Fig. 3E) [25, 26].

Specifically, once the suspected area is identified, measurements are carried out by placing ROI on the region of interest and on the portion of healthy gland for comparison, and typically, a highly significant PCa (ISUP grade group $\geq 2$ and/or Gleason score $\geq 7$ ) shows a highly suggestive contrast dynamics consisting of a high and rapid
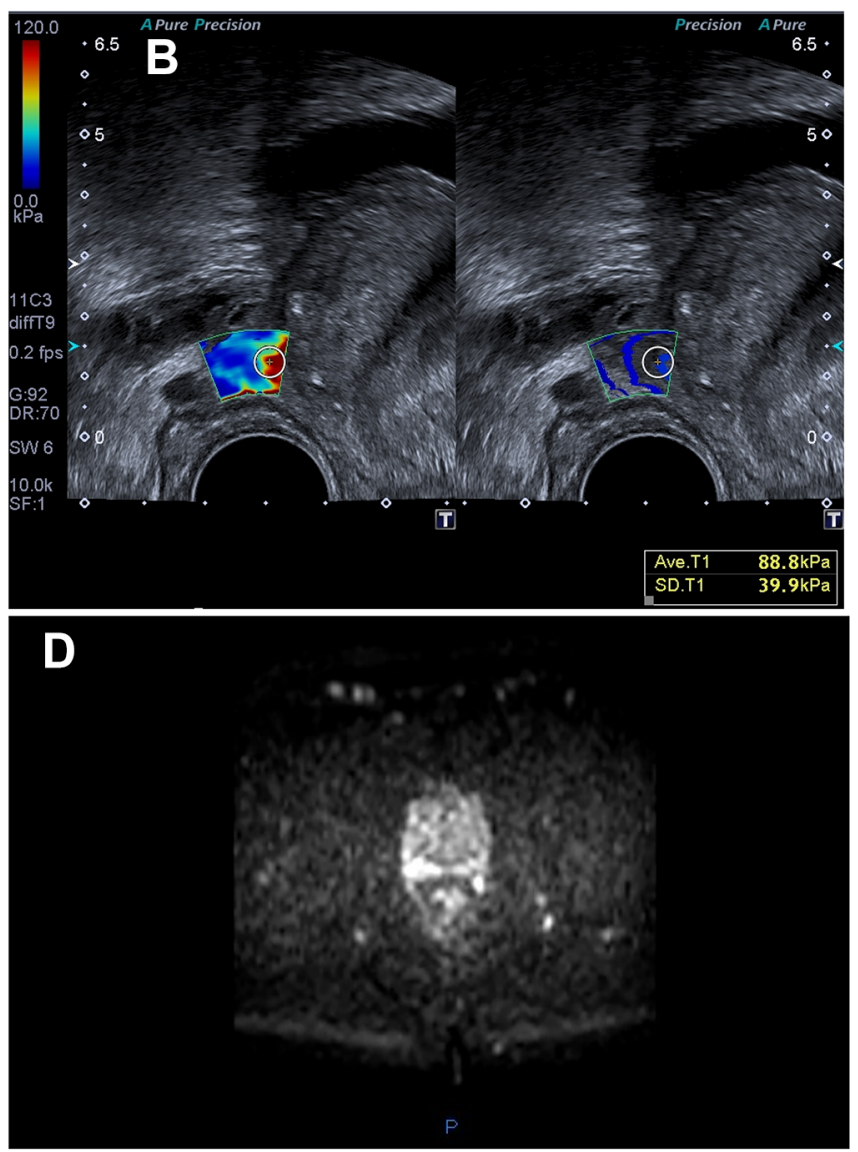

biopsy for conclusive diagnosis, has been confirmed by mp-MRI as well: red arrow in $\mathbf{C}$ shows a hypointense round-shape nodule, with slight extracapsular extension (ECE), showing restricted diffusion on diffusion-weighted imaging (DWI) (D) and apparent diffusion coefficient (ADC) map (E), with early intense hyperenhancement after contrast medium intravenous administration (F) (Color figure online) 
Fig. 4 continued
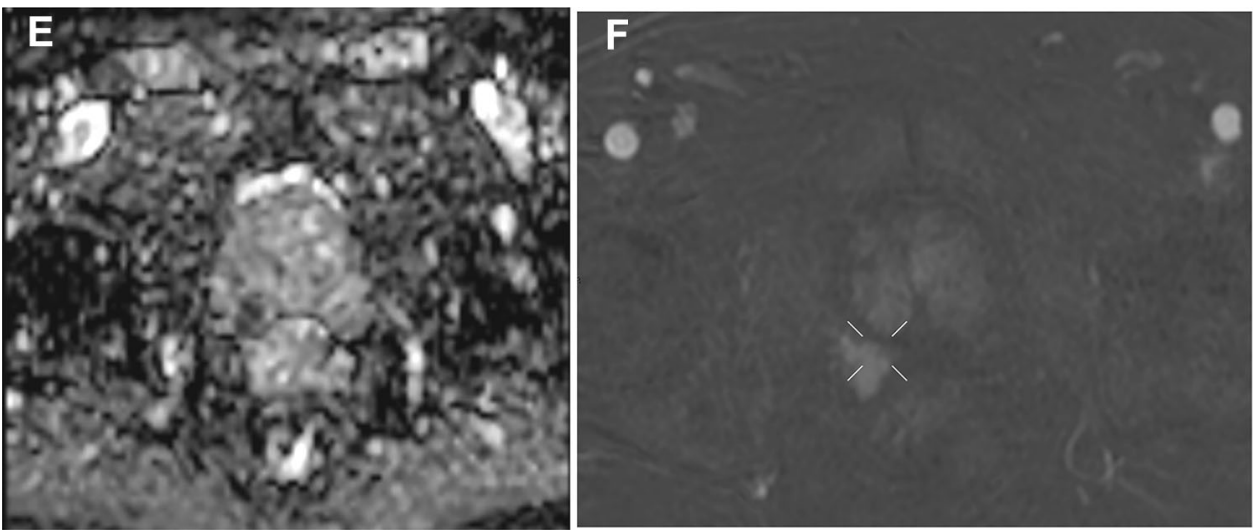

enhancement peak with a duration of less than $20 \mathrm{~s}$ from the injection of ultrasound contrast medium (Fig. 3E) [27].

Furthermore, since the described technique is strictly operator dependent, since the identification of the suspected lesion on which to place the ROI is related to the human eye and therefore susceptible to error, there is the possibility of editing enhancement curves on the entire prostate gland by letting the proper software to highlight the area with abnormal enhancement that will, of course, undergo a biopsy [28]. In this regard, the just described "Parametric Dispersion CEUS" seems to be more complete, safe, and reliable (with a sensitivity of $91 \%$, a specificity of $56 \%$, a positive predictive value of $57 \%$, and negative of $90 \%$ ), compared to CEUS alone (with a sensitivity of $73 \%$, a specificity of $58 \%$, a positive predictive value of $50 \%$, and negative of $79 \%$ ) [29].

\section{Prostate TRUS Elastography}

It is now widely known, especially in clinical practice that most prostate carcinomas have a harder consistency, by definition "ligneous," than the physiological glandular tissue, which allow their identification through digit-rectal examination (DRE) [30].

The greater rigidity of neoplastic tissue compared to healthy tissue is closely related to a series of changes that alter the architecture of the gland, such as the increase in cell density, the stromal reaction with collagen deposition in the periprostatic tissue, as well as the increase in microvascularization [31, 32]. These tissue properties and characteristics are not easily evaluated with traditional ultrasound imaging methods, such as the B-mode and Doppler mode described above, which do not provide information about in vivo prostatic elastic properties and, therefore, require measurements obtainable by means of elastography.

Prostate cancer, however, is not the only condition related to tissue and prostate gland elasticity variations: always remember that "not all cancers are stiff and not all stiff lesions are cancers" [8•]. It is not uncommon, in fact, that the presence of intraparenchymal calcifications or benign prostatic hyperplasia $(\mathrm{BPH})$ are responsible for an increase in the stiffness of the gland and, therefore, for false positives. It is, therefore, necessary to pay close attention during the execution of elastography to the patient's history, to any collateral findings (e.g., the involvement of extra-capsular tissues), to the degree of rigidity (closely related and directly proportional to Gleason score and degree of disease) [33] and, last but not least, to the portion of the gland being examined, not forgetting that while $\mathrm{BPH}$ is more frequent in the peripheral zone (PZ), carcinoma often affects the TZ [34].

In order to perform a full and as diagnostic as possible ultrasound examination, elastography should be performed after measuring the volume of the entire prostate gland with standard ultrasound on the longitudinal and axial planes, after having carefully inspected the periprostatic space and, where appropriate, after identification of the suspected area on which to focus attention [8•]. This last aspect, however, is by no means the "conditio sine qua non" for making elastography, since it has as its main advantage not only the characterization capacity of a suspected prostate lesion based on the degree of elasticity/ stiffness, but also especially to identify prostate lesions not visible to conventional TRUS without knowledge of the tumor site a priori [19, 35].

Elastography can be performed in two different ways: strain elastography (SE), which directly measures the tissue's stiffness and its ability to deform by means of a mechanical pulse, and shear-wave elastography (SWE) which measures the capacity of the sound wave to propagate within a tissue based on its elasticity [36].

\section{Strain Elastography (SE)}

The SE consists of the execution of continuous compression-decompression cycles of the gland by means of an 
endorectal probe, followed by a colorimetric map, the "elastogram," superimposable to the B-mode ultrasound images and showing the different degrees of elasticity/ stiffness of the various parts of the gland (Fig. 3C). In general, the most rigid and low-deformable tissues are encoded in blue, in contrast to the red color indicative of a major glandular elasticity, but other color maps may be used $[37,38]$. In order to obtain results that are as little as possible affected by the manual skill of the operator, therefore, by the presence of artifacts, it is essential that the pressure on the prostate is uniform and homogeneous, applied with the same intensity at all points of the gland without causing its slippage and, for this purpose, a waterfilled balloon is placed between the probe and the anterior wall of the rectum [39]. The region of interest (ROI) during the execution of the SE is extended to the whole prostate gland and partially to the surrounding tissue, which allows to generate the elastogram with ease by comparing the contiguous frames in a direct way, in which interpretation remains, however, strictly subjective and related to the operator's experience. In fact, although several recent studies have shown that the SE is more sensitive and specific than B-mode, see for example, the Aboumarzouk et al. meta-analysis [36] which showed percentages respectively in the range of $71-82 \%$ and $60-95 \%$ confirmed by radical prostatectomy histological examination, or even a retrospective study of Pozzi et al. [35] on a cohort of 460 patients which demonstrated how the diagnostic accuracy of US imaging increases significantly by associating SE with B-mode with an $80 \%$ sensitivity and a negative predictive value of $87 \%$, it should be borne in mind that the SE does not offer a quantitative and objective measurement of pathological prostatic tissue.

In the elastogram map, in fact, the color/stiffness scale is automatically distributed from the softest region to the most rigid one included in the ROI and the intermediate value obtained from the ratio between the values of the two regions remains, however, semi-quantitative and limited.

Therefore, if the limits of interpretation are added to those of execution, the SE does not prove to be at all superior to the prostate biopsy, in the identification of carcinomas, especially if small; at the most, it could be used as an aid in the execution of biopsy without, however, being used as a decision tool to rule out a prostate cancer bypassing biopsy [40, 41].

\section{Shear-Wave Elastography (SWE)}

While the SE requires active intervention and expert manual skills of the clinical operator, on the contrary, the SWE is in some ways much less operator dependent. The principle on which it is based, in fact, is the ability of ultrasound to propagate through a biological tissue, with a higher or lower speed depending on the degree of elasticity of the tissue itself that opposes more or less resistance [42]. Therefore, the compression-decompression cycles of the $\mathrm{SE}$, on the contrary, are considered an unfavorable element to the execution of a correct SWE, in which attempts are made to avoid as much compressive forces as possible on the examined tissue, which would be responsible for incorrect artifacts and measurements. It is, therefore, clear how often it is difficult to avoid compressing the prostate by performing a TRUS with SWE technique, especially if you are faced with a gland increased in volume and protruding through the anterior wall of the rectum, with the peripheral zone (PZ) appearing more rigid than it really is, because it is adjacent to the probe [43, 44].

In the prostate SWE, a beam of ultrasound, generated by an end-fire endocavitary probe, propagates transversely to the transducer providing a dynamic measurement, quantitative and real-time parenchymal rigidity based on speed $(\mathrm{m} / \mathrm{s})$ at which sound waves travel: the stiffer the tissue interrogated, the higher the speed of propagation of the ultrasonic beam.

In this way, the SWE is useful to calculate the so-called "modulus of elasticity" or "Young's modulus" (in kPa), as a direct measure of tissue rigidity, visually represented by a colorimetric map superimposed on the B-mode ultrasound images, similar to the SE. In the case of the SWE, however, the legend of colors is completely reversed: a rigid and, therefore, suspicious area in the prostatic tissue is encoded by the red color, while soft and physiological tissues are represented in blue (Fig. 4A, B) [45].

The commonly obtained parameters in the execution of the SWE are represented by elasticity values expressed in terms of average, standard deviation, minimum and maximum values of each individual ROI (also called "Quantitative box" or "Q box") scanned by analyzing the entire prostate gland from the apex to the base. In this way, it is possible not only to obtain an absolute quantitative measurement of tissue elasticity in the context of a highly suspected area, identified by B-mode or other imaging techniques, but also to detect ex-novo rigid lesions highly suggestive for carcinoma to subject to diagnostic deepening, for example, with a target biopsy under guide SWE $[8 \bullet$.

Being a fairly recent ultrasound technique, studies about its diagnostic usefulness are not so numerous, but each of them shows that prostate cancer has significantly higher stiffness values than the normal gland or then a benign lesion (p 0.002) [43]. In each clinical trial, different "stiffness cut-offs" have been established above which the suspicion of being faced with a malignant lesion grows in a way. Correas et al., for example, in a study of 184 patients, established an elasticity threshold of 35-37 $\mathrm{kPa}$ [34], unlike Boehm et al. which placed the threshold at a higher 
elasticity value of approximately $50 \mathrm{kPa}$ [46]. However, apart from these differences, high percentages (over $70-80 \%$ ) have always been found in terms of diagnostic accuracy, sensitivity, specificity, and negative predictive value, which confirmed the correlation between tissue rigidity and malignancy, which was always confirmed by the outcome of the histological examination [47]. In a study of 50 patients undergoing SWE-guided target biopsy on highly suspicious lesions, Ahmad et al. report a specificity of $88 \%$ and a sensitivity of $90 \%$ in patients with PSA less than $20 \mathrm{mg} / \mathrm{L}$ and much higher values, both of about $93 \%$, for PSA above $20 \mathrm{mg} / \mathrm{L}$. These data, together with the SE, confirm the existence of an even stronger correlation between glandular stiffness and prostate cancer, with a direct proportional relationship to the Gleason score and the degree of disease [48].

In the execution of a SWE elastography, although it is not susceptible to "external" variables such as the operator's manual skills, it is good to keep in mind the interindividual variability of patients and the anatomicfunctional modifications, even para-physiological, which the prostatic gland moves towards. Younger patients, whose prostate is not affected by benign prostatic hyperplasia (BPH) nor affected by degenerative senile sclerotic processes, peripheral zone (PZ), and central zone (CZ) have commonly a soft consistency and with mean stiffness values not exceeding $20-25 \mathrm{kPa}$, unlike the TZ whose rigidity is highly variable, due to its intrinsic tissue heterogeneity, and may even exceed $70 \mathrm{kPa}$, to reach even higher values as the gland develops a BPH $[49,50]$. The PZ remains, however, the most "reliable" portion of the gland in the evaluation with SWE, since it is inherently less rigid, less heterogeneous, and less susceptible to signal attenuation.

Thus, if, on the one hand, the SE proves to have numerous limits, either from an executive point of view, either from an interpretative one, on the other hand, the SWE seems to be on a higher step, proving to have an excellent reproducibility and low inter-observer variability especially if managed by expert hands.

\section{Multiparametric Ultrasound}

Like the mp-MRI, in recent years, we hear more and more often about multiparametric ultrasound (mp-US), but what is it really about? We know perfectly well that, unlike MR imaging, B-mode US does not have an intrinsic multiparametric properties that allow us to carry out an organ evaluation at 360 . However, it is thanks to the integration of classic grayscale imaging with specific US techniques such as the study of flows with the CDUS and PDUS, semi- quantitative analysis of tissue elasticity/stiffness with elastography and detection of a suspected lesion with CEUS, which allows us to get closer and closer to the concept of "multiparametric technique" [51].

One of the possible ways to exploit these ultrasound technologies in the context of the diagnosis of $\mathrm{PCa}$ is to obtain an integrated US-MR imaging dedicated to patients eligible for a target biopsy. The latter, in fact, are first subjected to prostatic mp-MRI, not only to assess the severity and the extent of the disease, undoubtedly important for therapeutic planning, but also to identify in a targeted way the highly suspected prostate lesion candidate for biopsy. The study of the prostate with US consists of an evaluation of the gland from a volumetric point of view, with axial and sagittal plane scans with B-mode US, corroborated by Doppler imaging and elastography to identify further possible suspicious lesions, in the face of which CEUS removes all possible doubts. In the end, it is possible to obtain an "MRI-TRUS fusion map," which highlights all prostatic lesions suspected for PCa candidate for biopsy, according to a precise risk stratification [6].

MRI-TRUS fusion, therefore, combines the iconographic and diagnostic advantages of MRI with the versatility of TRUS, and it is particularly useful in cases where the suspect PCa is identified only with mp-MRI to turn any doubt into certainty [52].

The future techniques of mp-US targeting involve the use of new developments in the field of Artificial Intelligence (AI) with deep-learning algorithms that will allow to combine functional and molecular parameters for the ultrasound detection of PCa. Computerized TRUS (CTRUS), for example, is an innovative system based on the use of artificial neural networks (ANN) to obtain a quantitative analysis of B-mode US imaging.

A work by Loch et al. conducted on a cohort of 132 patients, negative for $\mathrm{PCa}$ at systematic prostate biopsy and subsequently subjected to target biopsy with C-TRUS, showed the effectiveness of this technology in detecting PCa in $50 \%$ of patients, of which $45 \%$ with Gleason score 6 or less [53].

Similar situation in a study by Strunk et al., with a group of 20 patients with prostatic lesions identified by C-TRUS and highly suspicious for malignancy, 11 obtained histological confirmation of PCa after target biopsy [52].

\section{Conclusion}

Nowadays, the US has made great strides in the field of diagnostic and interventional radiology, especially thanks to the innovative ultrasound techniques recently developed and increasingly widely used. 
In particular, in the field of urological diagnostics, US technology appears increasingly accurate and more sensitive in terms of detection of PCa, US-guided target biopsy, and localized treatments. This is made possible thanks to specific US techniques, such as CEUS and elastography (SE and SWE) which, together with the classical US imaging of TRUS and CDUS/PDUS, allow to carry out a more complete organ study, including diagnostic deepening with biopsy of suspicious lesions, allowing us to talk about multiparametric US.

A pioneering study combining all modern US techniques, in order to assess their combined performance, was conducted by the Germans Brock et al. in 2013 [53]. They submitted to mp-US examination 100 patients candidate for radical prostatectomy and, therefore, with confirmed diagnosis of PCa. The lesions suspected for PCa were first identified with elastography and CEUS and, subsequently, histological confirmation was obtained on the samples identified by US imaging and obtained after prostatectomy [51]. If elastography alone showed a specificity value of $74 \%$ and a sensitivity of $49 \%$, the latter probably justified by the presence of small insignificant cancerous foci not detectable to US, the addition of CEUS in combination revealed an $89.7 \%$ probability of cancer detection.

The quality and quantity of studies available to date make, however, the experience in the field sufficiently poor, leaving still undefined the role of mp-US, both as a guide in the biopsy target as a diagnostic tool that can integrate the mp-MRI, but not obviate its utility to the point of replace it.

Nevertheless, the results obtained seem very promising and herald the potential for an imaging-based approach to prostate cancer management much more accessible to the wider community.

Funding Open access funding provided by Università di Foggia within the CRUI-CARE Agreement.

Open Access This article is licensed under a Creative Commons Attribution 4.0 International License, which permits use, sharing, adaptation, distribution and reproduction in any medium or format, as long as you give appropriate credit to the original author(s) and the source, provide a link to the Creative Commons licence, and indicate if changes were made. The images or other third party material in this article are included in the article's Creative Commons licence, unless indicated otherwise in a credit line to the material. If material is not included in the article's Creative Commons licence and your intended use is not permitted by statutory regulation or exceeds the permitted use, you will need to obtain permission directly from the copyright holder. To view a copy of this licence, visit http://creativecommons. org/licenses/by/4.0/.

\section{References}

Papers of particular interest, published recently, have been highlighted as:

- Of importance

•• Of major importance

1. Mottet N, et al. EAU-ESTRO-SIOG guidelines on prostate cancer. Part 1: Screening, diagnosis, and local treatment with curative intent. EurUrol. 2016. https://doi.org/10.1016/j.eururo. 2016.08.003.

2. Carpagnano FA, Eusebi L, Tupputi U, et al. Multiparametric MRI: local staging of prostate cancer. Curr Radiol Rep. 2020;8:27. https://doi.org/10.1007/s40134-020-00374-y.

3. Chen FK, de CastroAbreu AL, Palmer SL. Utility of ultrasound in the diagnosis, treatment, and follow-up of prostate cancer: state of the art. J Nucl Med. 2016;57(Suppl 3):13S-18S.

4. Loeb S, Bjurlin MA, Nicholson J, et al. Overdiagnosis and overtreatment of prostate cancer. Eur Urol. 2014;65:1046-55.

5. Litwin MS, Tan HJ. The diagnosis and treatment of prostate cancer: a review. JAMA. 2017;317:2532e42. https://doi.org/10. 1001/jama.2017.7248.

6. Ventrella E, Eusebi L, Carpagnano FA, et al. Multiparametric MRI of prostate cancer: recent advances. Curr Radiol Rep. 2020;8:19. https://doi.org/10.1007/s40134-020-00363-1.

7. $\bullet$ Grey A, Ahmed HU. Multiparametric ultrasound in the diagnosis of prostate cancer. Curr Opin Urol. Pioneering work about all the modern ultrasound modalities to consider their combined performance for a multiparametric approach. 2016; 26(1):114-119.

8. • Correas JM, Halpern EJ, Barr RG, et al. Advanced ultrasound in the diagnosis of prostate cancer. World J Urol. 2020. Complete and exhaustive works on the multidisciplinary approach to prostate cancer diagnosis. https://doi.org/10.1007/s00345-02003193-0

9. Ganie FA, Wanie MS, Ganie SA, et al. Correlation of transrectal ultrasonographic findings with histopathology in prostatic cancer [abstract]. J Educ Health Promot. 2014;3:38.

10. •Hricak H, Choyke PL, Eberhardt SC, Leibel SA, Scardino PT. Imaging prostate cancer: a multidisciplinary perspective. Radiology. Complete and exhaustive works on the multidisciplinary approach to prostate cancer diagnosis. 2007;243:28-53.

11. Lughezzani G, Saita A, Lazzeri M, et al. Comparison of the diagnostic accuracy of micro-ultrasound and magnetic resonance imaging/ultrasound fusion targeted biopsies for the diagnosis of clinically significant prostate cancer. Eur Urol Oncol. 2019;2:329-32. https://doi.org/10.1016/j.euo.2018.10.001.

12. Sauvain JL, Sauvain E, Rohmer P, et al. Value of transrectal power Doppler sonography in the detection of low-risk prostate cancers. Diagn Interv Imaging. 2013;94:60-7.

13. Halpern EJ, Frauscher F, Strup SE, et al. Prostate: high-frequency Doppler US imaging for cancer detection. Radiology. 2002;225:71-7.

14. Cheng S, Rifkin MD. Color Doppler imaging of the prostate: important adjunct to endorectal ultrasound of the prostate in the diagnosis of prostate cancer. Ultrasound. 2001;17:185-9.

15. Erbersdobler A, Isbarn H, Dix K, et al. Prognostic value of microvessel density in prostate cancer: a tissue microarray study. World J Urol. 2010;28:687-92.

16. Ezquer A, Ortega Hrescak MC, Sanagua C, et al. Transrectal Doppler ultrasound during prostate biopsy: clinical utility and limitations. Actas Urol Esp. 2015;39:13-9. 
17. Halpern EJ, Frauscher F, Forsberg F, et al. High-frequency Doppler US of the prostate: effect of patient position. Radiology. 2002;222:634-9.

18. Leen E, Averkiou M, Arditi M, et al. Dynamic contrast enhanced ultrasound assessment of the vascular effects of novel therapeutics in early stage trials. Eur Radiol. 2012;22:1442-50.

19. Sidhu PS, Cantisani V, Dietrich CF, et al. The EFSUMB guidelines and recommendations for the clinical practice of con-trastenhanced ultrasound (CEUS) in non-hepatic applications: update 2017 (long version). Ultraschall Med. 2018;39:e2-44. https://doi. org/10.1055/a-0586-1107.

20. Turco S, Frinking P, Wildeboer R, et al. Contrast-enhanced ultrasound quantification: from kinetic modeling to machine learning. Ultrasound Med Biol. 2020. https://doi.org/10.1016/j. ultrasmedbio.2019.11.008.

21. Zhao HX, Xia CX, Yin HX, et al. The value and limitations of contrast-enhanced transrectal ultrasonography for the detection of prostate cancer. Eur J Radiol. 2013;82:e641-7.

22. Harvey CJ, Pilcher J, Richenberg J, Patel U, Frauscher F. Applications of trans-rectal ultrasound in prostate cancer. $\mathrm{Br} \mathbf{J}$ Radiol. 2012;85(suppl):S3-17.

23. Li H, Xia J, Xie S, et al. Prostate cancer: a comparison of the diagnostic performance of transrectal ultrasound versus contrast enhanced transrectal ultrasound in different clinical characteristics. Int J Clin Exp Med. 2015;8:21428-34.

24. Roy C, Buy X, Lang H, et al. Contrast enhanced color Doppler endorectal sonography of prostate: efficiency for detecting peripheral zone tumors and role for biopsy procedure. J Urol. 2003;170:69-72. https://doi.org/10.1097/01.ju.0000072342. 01573.8d.

25. Zhang M, Tang J, Luo $\mathrm{Y}$, et al. Diagnostic performance of multiparametric transrectal ultrasound in localized prostate cancer: a comparative study with magnetic resonance imaging. J Ultrasound Med Off $\mathrm{J}$ Am Inst Ultrasound Med. 2019;38:1823-30. https://doi.org/10.1002/jum.14878.

26. Apfelbeck M, Chaloupka M, Schlenker B, et al. Follow- up after focal therapy of the prostate with high intensity focused ultrasound (HIFU) using contrast enhanced ultrasound (CEUS) in combination with MRI image fusion. Clin Hemorheol Microcirc. 2019;73:135-43. https://doi.org/10.3233/CH-199222.

27. Maxeiner A, Fischer T, Schwabe J, et al. (2019) Contrastenhanced ultrasound (CEUS) and quantitative perfusion analysis in patients with suspicion for prostate cancer. Ultraschall Med Stuttg Ger. 1980;40:340-8. https://doi.org/10.1055/a-0594-2093.

28. Yunkai Z, Yaqing C, Jun J, et al. Comparison of contrastenhanced ultrasound targeted biopsy versus standard systematic biopsy for clinically significant prostate cancer detection: results of a prospective cohort study with 1024 patients. World J Urol. 2019;37:805-11. https://doi.org/10.1007/s00345-018-2441-1.

29. Postema AW, Frinking PJA, Smeenge M, et al. Dynamic contrast-enhanced ultrasound parametric imaging for the detection of prostate cancer. BJU Int. 2016;117:598-603. https://doi.org/10. 1111/bju.13116.

30. Good DW, Stewart GD, Hammer S, et al. Elasticity as a biomarker for prostate cancer: a systematic review. BJU Int. 2014;113:523-34.

31. Miyagawa T, Tsutsumi M, Matsumura $\mathrm{T}$, et al. Real-time elastography for the diagnosis of prostate cancer: evaluation of elastographic moving images. Jpn J Clin Oncol. 2009;39:394-8. https://doi.org/10.1093/jjco/hyp026.

32. Chou R, Croswell JM, Dana T, Bougatsos C, Blazina I, Fu R, Gleitsmann K, Koenig HC, Lam C, Maltz A. Screening for prostate cancer: a review of the evidence for the US Preventive Services Task Force. Ann Intern Med. 2011;155(11):762-71.

33. Barr RG, Cosgrove D, Brock M, et al. WFUMB guidelines and recommendations on the clinical use of ultrasound elastography: part 5. Prostate. Ultrasound Med Biol. 2017;43:27-48. https://doi. org/10.1016/j.ultrasmedbio.2016.06.020.

34. Correas JM, Tissier AM, Khairoune A, et al. Ultrasound elastography of the prostate: state of the art. Diagn Interv Imaging. 2013;94:551-60.

35. Pozzi E, Mantica G, Gastaldi C, et al. The role of the elastography in the diagnosis of prostate cancer: a retrospective study on 460 patients. Arch Ital Urol Androl. 2012;84:151-4.

36. Aboumarzouk OM, Ogston S, Huang Z, et al. Diagnostic accuracy of transrectal elastosonography imaging for the diagnosis of prostate cancer: a systematic re-view and meta-analysis. BJU Int. 2012;110:1414-23.

37. Junker D, Schäfer G, Aigner F, et al. Potentials and limitations of real-time elastography for prostate cancer detection: a wholemount step section analysis. Sci World J. 2012;2012:193213. https://doi.org/10.1100/2012/193213.

38. Zhang B, Ma X, Zhan W, et al. Real-time elastography in the diagnosis of patients suspected of having prostate cancer: a metaanalysis. Ultrasound Med Biol. 2014;40:1400-7.

39. Tsutsumi M, Miyagawa T, Matsumura T, et al. Real-time balloon inflation elastography for prostate cancer detection and initial evaluation of clinicopathologic analysis. AJR Am J Roentgenol. 2010;194:W471-476. https://doi.org/10.2214/AJR.09.3301.

40. Brock M, von Bodman C, Palisaar RJ, et al. The impact of realtime elastography guiding a systematic prostate biopsy to improve cancer detection rate: a prospective study of 353 patients. J Urol. 2012;187:2039-43. https://doi.org/10.1016/j. juro.2012.01.063.

41. Wang R, Chen J-J, Hu B. Transrectal real-time elastographyguided transperineal prostate biopsy as an improved tool for prostate cancer diagnosis. Int J Clin Exp Med. 2015;8:6522-9.

42. Ji Y, Ruan L, Ren W, et al. Stiffness of prostate gland measured by transrectal real-time shear wave elastography for detection of prostate cancer: a feasibility study. $\mathrm{Br} \mathrm{J}$ Radiol. 2019;92:20180970. https://doi.org/10.1259/bjr.20180970.

43. Woo S, Suh CH, Kim SY, et al. Shear-wave elastography for detection of prostate cancer: a systematic review and diagnostic meta-analysis. AJR Am J Roentgenol. 2017;209:806-14. https:// doi.org/10.2214/AJR.17.18056.

44. Porsch M, Wendler JJ, Liehr UB, et al. New aspects in shearwave elastography of prostate cancer. J Ultrason. 2015;15:5-14.

45. Boehm K, Salomon G, Beyer B, et al. Shear wave elastography for localization of prostate cancer lesions and assessment of elasticity thresholds: implications for targeted biopsies and active surveillance protocols. J Urol. 2015;193:794-800.

46. Boehm K, Budäus L, Tennstedt $P$, et al. Prediction of significant prostate cancer at prostate biopsy and per core detection rate of targeted and systematic biopsies using real-time shear wave elastography. Urol Int. 2015;95:189-96. https://doi.org/10.1159/ 000431233.

47. Yang Y, Zhao X, Zhao X, et al. Value of shear wave elastography for diagnosis of primary prostate cancer: a systematic review and meta-analysis. Med Ultrason. 2019;21:382-8. https://doi.org/10. $11152 / \mathrm{mu}-2051$

48. Ahmad S, Cao R, Varghese T, Bidaut L, Nabi G. Transrectal quantitative shear wave elastography in the detection and characterisation of prostate cancer. Surg Endosc. 2013;27:3280-7.

49. Kratzenberg J, Salomon G, Tennstedt P, et al. Prostate cancer rates in patients with initially negative elastography-targeted biopsy vs. systematic biopsy. World J Urol. 2018;36:623-8. https://doi.org/10.1007/s00345-018-2178-x.

50. Sarkar S, Das S. A review of imaging methods for prostate cancer detection. Biomed Eng Comput Biol. 2016;7:1-15.

51. Brock M, Eggert T, Palisaar RJ, et al. Multiparametric ultrasound of the prostate: adding contrast enhanced ultrasound to real-time 
elastography to detect histopathologically confirmed cancer. J Urol. 2013;189:93-8.

52. Strunk T, Decker G, Willinek W, et al. Combination of C-TRUS with multi- parametric MRI: potential for improving detection of prostate cancer. World J Urol. 2014;32:335-9.

53. Loch T. Computerized transrectal ultrasound (C-TRUS) of the prostate: detection of cancer in patients with multiple negative systematic random biopsies. World J Urol 2007; 25:375-380. Erratum in: World J Urol. 2013 Aug; 31(4):1019.

Publisher's Note Springer Nature remains neutral with regard to jurisdictional claims in published maps and institutional affiliations. 